\title{
DE JURADOS Y ARQUITECTOS: IDEAS SOBRE LOS CONCURSOS
}

\section{JURIES AND ARCHITECTS: IDEAS ON COMPETITIONS}

Jesús Rojo Carrero

RESUMEN Los concursos de ideas de arquitectura fallados por jurados participados por profesionales cualificados prestigian a as entidades que los promueven y a los arquitectos premiados. Pero tambien entrañan aspectos polémicos que ponen de relieve dicación pous yue pocas veces son analizados con actitud critica. Pese a todo, los arquitectos prefieren este sistema de adju-

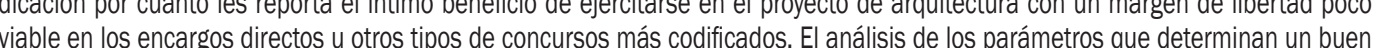

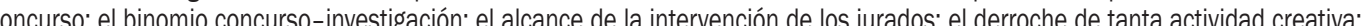
el trabaji no remunerado de los concursantes... En este artículo avanzamos una reflexión sobre algunos de estos temas a partir de PALABRAS

SUMMARY Competitions of architectural ideas that are judged by professionally qualified juries bring prestige to the institutions that promote them and to the award-winning architects. However, controversial aspects are also involved that highilight the weaknes-
ses of these competitions and which are rarely analysed critically. None the less, architects prefer this award system because of the concomitant benefit of practicing in architectural projects, with a margin of freedom that is unfeasible in direct commissions or other more codfied competitions. The analysis of the parameters which determine a good competition involves: the competition-research

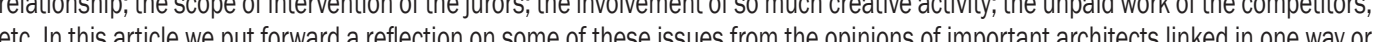

Fich

Pesona de contacto / Corresponding author: jesusrojo@arquired.es. Arquitecto.
"El concurso es una oportunidad para una investigación muy limpia: ese es su lado bueno. Su lado negativo sería la ausencia de cliente y la falta de interacción con éste; y su lado peor, tirar a la basura tanta investigación"1. Joan Roig i Duran (Batlle \& Roig Arquitectes). entro de la producción arquitectónica los conide soluciones magistrales tes complejas y singulares. La oportunidad de celebrarlos nace por decirlo de un modo simpley rotundo, de una esencial pusencia de ideas: surgen con la intención de superar el estancamiento de modelos y procedimienos contrastados que en la ocasión planteada, no parecen plenamente satisfactorios La concurrencia competitiva de los profesionales fuerza la exploración de caminos inédilos o la emergencia de nuevos talentos. Los concursos de deas prestigian a promotores y concursantes, y el impacto de la solución premiada, la valía de las propuestas desestimadas o la repercusión social del proceso, marcan el carácter innovador y polémico que se les suele asociar y que es reconocible en ciertos hitos de la historia de la arquitectura: el concurso para el pabellón de la primera Exposición universal de Londres, en 1850; el organizado por el diario Chicago Tribune en 1922; en 1927 el de la sede en Ginebra para la Sociedad de Naciones; el concurso

para el Palacio de los Soviets de 1931; el concurso para la Casa de la Opera de Sidney de 1957; el centro Geor .

En el panorama reciente hemos vivido un periodo proIfico en concursos de arquitectura incremento patente en nuestro país tras la promulgación en 1995 de la Ley de Contratos de las Administraciones Públicas, adaptando la antigua Ley de Contratos del Estado a las Directivas Comunitarias de la Unión Europea y definiendo un marco regulador para todo tipo de contratos, además de los espećíicos para la obra pública² Los principios de transparencia, publicidad y libre concurrencia son de este modo codificados e incorporados al estado de derecho y desaparecen prácticamente las adjudicaciones directas que con criterios dispares, eran aún habituales casi veinte años después de nuestra incorporación a la nómina de las democracias occidentales.

De este modo, el tradicional concurso de ideas de arquitectura se ha visto absorbido por la praxis de la gestión pública, solapándose dos trayectorias que originalmente

1. Exposición Concursos de Arquitectura con participación de jurado, CPAJ 2002/2006. Video-entrevistas de la exposicion. Sevilla, enero de 2008. Junta de Andalucia; Consejería de Obras Publicasy Transporte.

2. Ley 13/1995, de 18 de mayo, de contratos con las Administraciones Públicas. Exposición de motivos. 1 Justificación de la nueva Ley. BOE: num. 119 de 19 
poco tenían en común: a la demanda de innovación y calidad de los concursos de arquitectura se ha yuxtapuesto la exigencia de garantías jurílicas del procedimiento administrativo. De tal manera que en este tránsito del concurso como opción voluntaria al concurso como imperativo legal, se producen desen
morosos conflictos.

morosos conflictos.
Salvo poco representativas excepciones, la escasa cultura general de nuestra sociedad en materia de arquilectura y la ocupación de la iniciativa privada en cuestiones muy alejadas de la creación arquitectónica, incluso hostiles a esta, ha concentrado el interés de los profesionales en la Administración como el cliente con el que poder ejercer en condiciones dignas su actividad y desaollar con cierto margen la innovación.

Con el nuevo marco normativo el arquitecto se enfrenla a la obligación de revalidar iterativamente su capacitaque se depuran en términos de solvencia administrativa, que se depuran en términos de solvencia aaministrativa económicay profesional. Esto viene a acelerar entre otras causas la roconversion de los anosanalos estudios de arquilectura, ajenos a los esquemas del mundo empresaral hasta el momento, en estructuras de producción más

Denro de la variedad reglamentada por la Ley nos centraremos en aquelos concursos en los que el fallo vie-

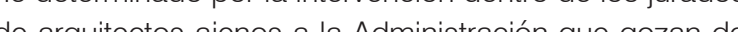
una solves ajos a con su presencia el procedimiento dándose por hecho aue las ideas por ellos pemiadas lo son por su calidad por suaciet p por su valor diferencial sobre as restantes soluciones tomadas en consideración.

Estas reflexiones nacen del desarrollo de una exposición retrospectiva de concursos de arquitectura celebrada

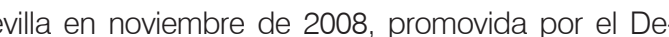
partamento de Fomento de la Arquitectura de la Junta de

Andalucía, para la cual se recabaron, a través de entrevistas grabadas en video, las opiniones de varios arquitectos nacionales inviderados con asiduidad en los concursos de ideas de arquitectura con jurado ${ }^{3}$. En cierto modo lo aqu expuesto responde a una época que pudiera apreciarse como tiempo ya pasado, dado el profundo panorama de crisis que la producción arquitectónica vive arrastrada por la crisis general del sector de la construcción, a la vez que a Administración casi ha abandonado su papel como promotor de obra pública, cuanto más de Arquitectura, estigmatizada en nuestros dilas por la opinión pública como algo caprichoso, prescindible, y viable sôlo desde la abundancia. En este estado de cosas, aún cuando es legalmente . elualibles, se opla preferentemente por procedimientos con requisitos tan in ilativos que la figura del arquitecto queda reducida a la de un facultativo con cobertura de un seguro, vención a nives de anonimato o incluso sometimiento. EL POR QUÉ DE LOS CONCURSOS DE IDEAS CON

\section{JURADOS CUALIFICADOS \\ (}

Apoco bien que esté organizado un concurso, en general cuena En muchos países cuando se han adoptado los sistemas de concursos, inmediatamente se ha notado una subida de la calidad media de la arquitectura que se produce". Antonio Oriz Gaćl (Cruzy Oriz Aravitectos)

Solo con analizar algunas experiencias próximas en e aoptarporun procedimientoncomplicadoycostoso opor os motivos por los que los profesionales responden de formatan sor Ya hemos apuntado que los prestigiosos concursos de Ya hemos apuntado que los prestiglosos concursos de mmediatas con las que acome un problema complejo

3. Exposición Concursos de Arrquitectura con participación de Jurados, CPA 2002/2006, inaugurada en Sevilla en enero de 2008, promovida por el Servicio de Arquitectura (Dpto. de Fomento de la Arquitectura) de la Consejerría de Obras Públicasy y Transporte de la Junta de Andalucía. Esta exposición itineróa a Granada Producción, guión y coordinación de las entrevistas: Julio Barreno Gutiérrez.

La mayoría de las citas recogidas en este earticulo están extraílas de las entrevistas grabadas en video para a dicha exposición. Alt tratarse de transcripciones de respuestas orales espontáneas a un guión de preguntas omitidas en la grabación, se ha adaptado la sintaxis y modificado algunas expresiones para hacer las citas más legibles y adecuadas a la expresión escrita, procurando siempre la máxima fidelidada a los contenidos y a la literalidadd de lo expresaddo por sus autores novedoso, o simplemente para forzar la respuesta original. Siendo esto así, el promotor público busca además legtimar la solución que se ensaye con la garantia de actuar de la forma más participativa y pública posible, llamando a escena a las mentes mejor preparadas, concursantes jurados, capaces de pensar y evaluar soluciones diversas y que estas sean presentadas a la ciudadanía de forma ilusionante. El éxito de la obra futura se adelanta a su propia construcción, y quedará avalado por la capacidad y altura profesional de los intervinientes, constatable en la representación virtual de la solución elegida. En el otro extremo el posible fracaso dejará diluida la responsabilidad entre tos actores implicados, quedando intacta la buena vol

Es un procedimiento impecable y democrático. Es una certeza histórica que cierta arquitectura ha estado siempre intimamente ligada al poder y se convierte en un valor probatorio de sus exitos, sobreviviendo a su propia vigencia. En el ejercicio de su responsabilidad polica, el gobernante rara vez se resiste a la seducción de

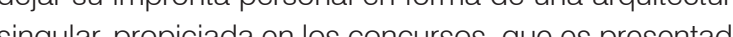
singuar, propiciada en los concusos, que es presentada younto

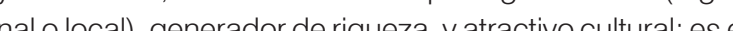
, milagro transformador del destino de la ciudad de Bilba operado por el elficio de Frank Gehn a finales del pasado siglo Pero es también una realidad que "no siempre una arquitectura de marca resuelve un problema y se cuenta para ciertas interenciones con un corto phazo: el de una legislatura Un problema profundo de arquitectura o urbanismo ha de resolverse en el largo plazo" "Carmen Imbernón Garća Secretaria General de EUROPAN-España).

En cuanto a los arquitectos, el concurso juzgado por En cuanto a los arquitectos, el colicurso juzgado por bilidad de conquistar un estatus desde el que poder eiecer la profesión a una escala de mayor interés y volumen y hacerlo además en condiciones de libertad, respeto e independencia inviables desde la praxis habitual de una profesión cada vez más masificada y devaluada socialmente. Es obvio por tanto que para el arquitecto el concurso de ideas con jurado es un objetivo en sí mismo, incluso

4. Un reciente ejemplo es el concurso restringido del Centro Nacional de Artes Visuales, promovido en 2008 por el Ministerio de Cultura (SEGIPSA), muy contestado por el Consejo Superior de los Colegios de Arquitectos de España y el propio Colegio de Madrid (Ver, por ejemplo, edición en papel de EL MUNDO de
14 de noviembre de 2008, p. 52)

cuando ese encargo, de lograrse, se vea envuelto en frusUnd se trata de una oportunidad

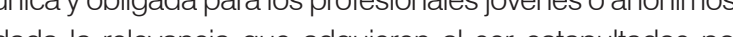
(ita expertos de primera linea. Aunque esturzo nó sea correspondido con un encargo firme o .

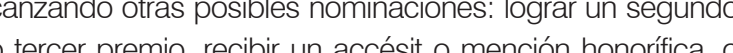
o asír recogido en las actas de los jurados, constituye un ser

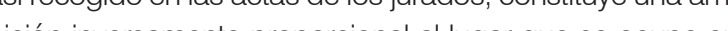
el ranking de aspirantes.

Agregar que la modalidad preferida por el arquitecto postulante, si restringida o abierta, va a depender de su mar chamo. Ningún arquitecto de prestigio admitira concursar en igualdad de oportunidades junto al novelo desconocido. e un lado porque estrifa núl consumir recursos en las que puede suponer no ganaras en favor de cold

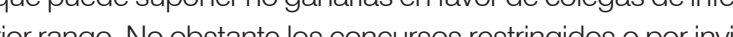
cín cuanto que la elección de un limitado elenco agravia un aún me profesionales no convocados, mezclándoos de falta de litimidad con las dudas solo suleglidat. Acerca de la poĺmica inherente a los concursos no abces, Jan Roig opina que "si se quere (que la intervencín la leve a cabo) un determinedo arquitecto de presjib la le permite sin más problemas: I Administracín sólo tiene que elaborar un dictamen diciendo que éste es (c) con veces se da en la que piensas que un concurso está quañalo" De algín modo un concurso de ideas con jurado y restringido a participantes invitados, entraña una cierta contradicción por cuanto se limita el concepto mismo de concurso: las ideas ceden protagonismo en favor de la marca de autor, por lo cual el procedimiento adquiee una dimensión más formal que de fondo dado que los arquitectos de alto nivel convocados asegurarín obras de interés arquitectónico aún cuando el 
LAS CLAVES DE UN BUEN CONCURSO arquitectura, un buen jurado es por lo menos tan importante como los buenos concursantes". Manuel de las Casas, Catedrático de Proyectos ETSAM, Politécnica de Madrid.
Desde la percepción del concursante, la valoración de los parámetros esenciales que deben garantizar la calidad undamentalmente en estos puntos:

- La elección de un jurado de profesionales cualificados, solventes y ecuánimes.

- La adopción idónea del modelo de concurso y la elaboración adecuada de las bases.

En lo relativo al jurado, parece ser opinión común que éste sea proporcionado en cuanto a su composición y competente en cuanto a la calidad de sus miembros Los concursantes demandan que las decisiones recaiprestigio, no vinculados al aparalo de la Administración Parece asi confiarse en un juicio estrictamente arquitectónico y libre de contaminaciones.

政 os jurados clebe haberuna mayoría de arqullectos (además

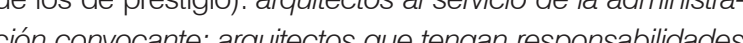
coin co coción. El poltico también debe estar $y$ tiene que saber

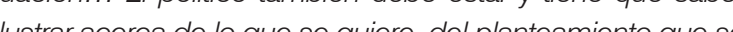

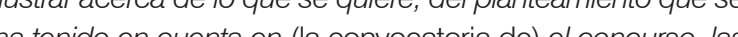

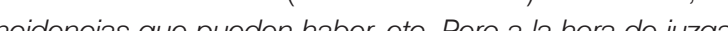
el promia el papelde los arquitectos es fundamenta".

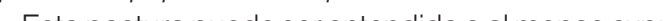

de forma más radical por cierto sector de la profesión

En palabras del arquitecto Ramón Fernández Alonso " propiedad deberí mantenerse al margen: tiene que dete gar en el jurado. Los jurados deben estar absolue deleintegrados por arquitectos que conozcan las necesidades que (el promotor) tiene a problemática objeto del concura - esta sería una labor extra que tendrí que ostablecer .... esta senta ma labor extra que tendría que establecer

Enado secundarios a nuestro se sitúa en lugares de-

adoptar criterios más objetivables que limiten en lo posible
"Para que un concurso sea realmente interesante a nivel de $y$ limpieza de un concurso de ideas con jurado se centran gan fundamentalmente en el criterio de los arquitectos de

apetencias personales o intereses coyunturales que desvien del jurado de la intención del concurso. No se puede pasar por alto que en la mayoría de los casos los miembros cualificados de un jurado son también arquitectos en activo y contendientes en otros concursos, y por tanto su

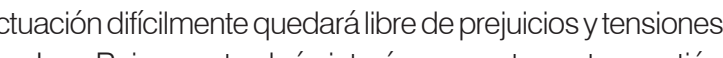
Joan Roig apunta algún interés por centrar esta cuestión Hay determinados arquitectos que se convierten en asiduos miembros de jurado y que van cambiando a lo largo del tiempo. No es una mala opción y no lo critico. ¿Qué ocurre con esto? Pues que nadie es aséptico: son figuras que imponen su qusto y su opinion determinante sobre ciertas cosas. Qui zás estos referentes debieran estar más mezclados (dentro mismo jurado) y así se diese pie a más discusión".

Por nuestra parte, y al margen de estas opiniones, nos atrevemos avanzar aquí alguno de los importantes deficilts estruclurales que encontramos en la actuación de - Pazo in y como están hoy concebidos:

- Plazo insuficiente para el examen de las propuestas. dato puón del número de concursantes presentados, este dato puede llegar a ser por símismo escandaloso. Podeexposición unas media minutos; según las propuestas hayan superado o no los primeros cortes seloctivos. Tambén se pueden ilustrar sesiones una sola jornada en las que han sido exaninas más de un contenar de trabajos Aunque se exime mucho laca

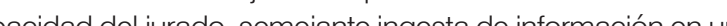
ter lo sión inevitable de errores de apreciaćn ola mudazade

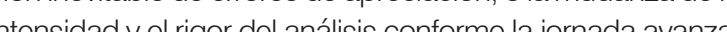
presionados por el limitado plazo para ever

- Escasa profundización del jurado en el conocimiento

de les bases y condiciones técnicas del concurso. Por

general, el concursante inscrito recibe una abundante docu mentación que ha de asimilar en sucesivos acercamientos problema planteado Sin embargo los técnicos de prestigio externos delosjurados apenassisoninformados convenienemente de estas bases en la misma sesión del fallo por lo que pueden llegar a desconocer en gran medida los objetvos y compromisos del promotor al eniuiciar las propuestas.

2002/2006: CD catálogo de la exposición. Sevilla, enero 2008.

6. Molina, Carlos: Arquitectura participativa. En Hechos, noticias, actualidad. Mayo 2007, № 21. Madrid: Hermandad Nacional de Arquitectos, pp. 42-46.
Estas deficiencias pueden llegar a degradar el trabajo de los concursantes, pero además también debilitan la autoridad del jurado, abocado a dictaminar con importantes carencias. Debilidades del sistema que son, por otra parte, bien conocidas por los aspirantes experimentados, que saben priorizar en sus trabajos la baza de la sorpresa, la expresión atractiva y el dominio de técnicas publicitarias. En palabras del arquitecto Josep Llinás y Carmona, "hay arquitectos que trabajan los concursos con una voluntad Clara de ganarlos utilizando sistemas infográficos y representativos que puedan deslumbrar a jurados poco ormados". Se reafirma implicitamente la importancia de un buen jurado como antidoto ante estas veleidades.

Pero el acierto de un jurado va a depender también de los medios a su disposición, de modo que aunando calidad proestonaloncalladde procedimiento, losconcursos podían salvar muchas de las ulleriores dificullades de gestion de los Se puede tombién planter polén Se puede también plantear la oportunidad de profesionalizar y revalorizar aún más los jurados, aspecto contovion pón profesiona con liva pencuanto a perdicla de perspectiva y concentracion de poder Sobre este paricular, se planteale la figura de los referee o comités de expertos rotativos habitules en otros campos del saber capaces de en condiciones no tan estresantes y desde el doble anonmato la producción cientifica especializada Para Luis Fer nández-Galiano Catedŕtico de proyectos de la ETSAM, Politécnica de Madridy director de las revistas Aqu Viva, AV Monografís y Arquitectura proyectos, el papel de wa, AV Montos ya existe: "es el que juegan los a pul cos estos expertos ya existe. "es el que juegan los arquitectos auncu no son ánimos sino muy públicos un papelmuy critico y decisorio en el desarrollo de los debates".

Como hemos introducido al principio de este punto el lugar común en las reflexiones de los arquitectos participantes parece residir en la creencia de que la categoría profesional de los miembros del jurado garantiza con suficiencia la calidad de un concurso. Sin menoscabo de esta dea, queremos hacer aquí énfasis en que a pesar de esa turales de un concurso pueden mermar sustantivamente capacidad del jurado, restar ecuanimidad al fallo e incidi muy negativamente en el servicio que este debe prestar concursantes, organizadores y a la ciudadanía en genera.
LA PARTICIPACIÓN CIUDADANA

Oro argumento a debate es la forma en la que, en aquelas inicialivas de allo inters general, ha de tener cabida la voz del ciudadano, bien con carácter consultivo, bien ro jurados populares con capacidad decisoria. La opinión mayoritaria rechaza estos estamentos por su falta de cualificacion y su potencial tendencia a sobre valorar lo anecdótico y superficial. Como mucho, las voces autorizadas más conciliadoras defienden que los jurados profesionales deberian por norma exponer didácticamente ante el gran público sus decisiones y valoraciones. Para Manuel de las Casas, el jurado debería siempre "darl la cara irente a los concursantes y frente a la sociedad a la que se esta respon dinndo" explcando y argumentando públicamente las propuestas premiadas. Los más reacios sostienen en cambio, que los cargos públicos son elegidos por el cruadano y, con esta responsabildad dmocralica, dede los jurados que ellos mismos convocan de los jurados que ellos mismos convocan. Lo contrario ca", en palabras del arquitecto Antonio Ortiz.

En opinión de Joan Roig "la participación ciudadan

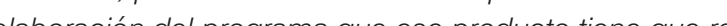
solver". Es afin a este pensamiento el Catedrático de Historia Teoría y Composición Arquitectónicas de la ETSA de Sevilla, Víctor Pérez Escolano, para el que "es cad vez más frecuente a proposición de ideas en el ma caco del proceso de participación pública en los phanes urbanístcos. Es ahi donde procede una cietta decantación popular de objetivos, pero nunca en la elección de propuestas ya de rango proyectual orientables por un supuesto gusto

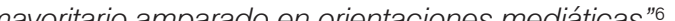

ANTES Y DESPUÉS DE LOS CONCURSOS Tras la experiencia acumulada debería también abordarse e hasta ahora es habitual, ésta empieza y acaba en las sesiones de deliberaciones y fallo del concurso, abandonando a su suerte a responsables públicos y premiados, o si debe implicarse de algún modo a lo largo de todo el proceso concursal, incluso en el seguimiento del proyecto y la obra Se tratarí de una nueva carga de responsabilidad para sjurados, perotaly comoafirma Ramón Fernández-Alonso "alabordel jurado siempreparece pequeña'éstadeberíen pezar cuando se comienza a elaborar las bases y terminari 
garantizando, tutelando la idea propuesta para que llegue construirse y no desampararla en ningún momento". Por su alta valoracion profesional los jurados externos deberían al menos tener voz para intervenir como consejeros y árbitros en las cotidianas fricciones entre el promolor y el arquitecto adjudicatario, controversias que tantos recursos públicos pueden consumir de manera estéril. Josep Llinás ilustra muy gráficamente una situación habitua tras los concursos: "En ocasiones los jurados les entregan los proyectos ganadores a instituciones con otro nivel, con otra escala. grandes proyectos que acaban en manos pequeñas. Estaría bien que (el jurado) hiciera un seguimiento para amparar el proceso hasta no sé bien qué momento.. Francisco Torres situa la responsabilidad entre las dos
partes afectadas: "Hay promotores que toman el resultado partes afectadas. Hay promotores que toman el resultado como un primer paso que se ha dado en una dirección y que hay que seguir en esa direccion con cierto rigor, y otros que lo ven como algo que les ha caído encimay aver como (lo reconverten. (Tras el fallo) se abre un proceso clentearquitecto en el que los dos tienen responsabilidades, y

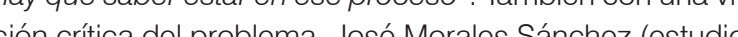
M CETSA Sevila), apuasta por "centrar aron pa proyectos plicación del jua en las miembros de badminitración pera que la gestín del projecto se haga de acuerdo con para que la gestion en lo ploción de un resultad Hay ugar para que las sugerencies al proyecto (elogido) se anoten en as actas del jurado y a

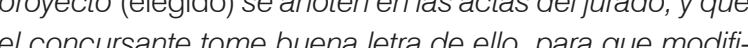
que algunas cicunstancis del proyecto que ha entegado. A veces se lega a produci lo contrario: se espera a sordina de la gestión para traicionar la idea del concurso".

Estas opiniones dibujan una realidad reconocible por

Equellos que se hayan visto envueltos en el desarrollo de proyectos y obras emanadas de concursos. Se trataría de un cambio brusco de discurso, difícil y doloromente asumible por los arquitectos concursantes: una valoración inicial, selectiva y decisoria sobre las propuestas desde el terreno casi exclusivo de la arquitectura on el momento del fallo del jurado; e inmediatamente después y al margen de los jurados y sus análisis, una gestión centrada de forma consenvadora en aspectos principatmente económicos y constructivos, desvinculada cuando no contraria a los parámetros que evaluaron su elección tensando el proceso con el objetivo de reconducir los proyectos a territorios mas convencionales y contrasta, banalizando las ideas premiadas.

No podemos dejar de traer a estas reflexiones algunos apuntes sobre aspectos que también van a marca el nivel de una convocatoria: la elección del modelo de concurso y la preparación de las bases.

En ambos temas puede jugar un papel importante la Secretaría Técnica elegida por el convocante y que según los casos desarrolla un papel diverso, según el promotor le asigne tan solo la tarea de garante del cumplimiento tormal del procedimiento administrativo, o le confiera la esponsabilidad de instruir intelectualmente el concurso Carmen Imbernón sitúa en el ámbito de las deliberaciores técl jurado a este personaje de reparto. "El secretaio técnico es la persona encargada de transmitir a todos objetivos proves que se han planteado. Es a quel que, al margon pro quén se han hla prode. Es aquel que, a dergn de la deción hovidual de delo Es nado miembro

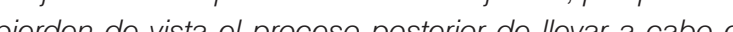
prover a cabo el

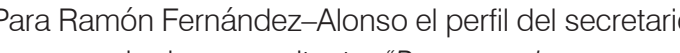
( Para te timpo su funcla persona que sepa traducir al lenguaje de la aruitectura as demandas de la propiedad Esta persona es el secretro técnico y es incustiturble". Efectivamente esta tarea de síntesis es trascendental. En caso contari, las incertidumbres que on ocasiones se tras adan a los concursantes son tan extensas, inconexas y de contenidos tan amplise, incluso incompetibles, que etconcursante se ve pronto tentado a la respuesta aventuraday poco ajustada

Una de las primeras decisiones que ha de abordar la Secretaría Técnica encarcada de montar la convocatoria será elegir su formato. El concurso de ideas de arquitectura con jurado tiene su expresión esencial en la convocatoria abierta, internacional y anónima con propuestas presentadas bajo lema en las que la identificación de autor es un asunto irrelevante en el proceso de selección de las ideas. En la versión más antagónica al espírtu de a libre concurrencia de ideas se situaría el concurso restringido por invitación expresa de los contendientes, en el que el anonimato de las propuestas es generalmente obviado ya que tan importante o más que la idea puede legar a ser el prestigio profesional y mediático de la firm que la presenta. Entre ambos modelos se han ensayado diversas hibridaciones: concursos en dos fases, en los que hay una primera selección de concursantes en virtud de su Curriculum Vitae o por el examen de propuestas nivel de ideas previas; o bien por una selección mixta con ambas modalidades (curriculum e ideas) en esa primera fase; concursos de propuestas avalados por empresas constructoras solventes que en cierta forma responden de la viabilidad de las mismas, concursos de proyectos casi ejeculivos buscando de nuevo el examen eficaze inmediato de la idea seleccionada; concursos de ideas $n$ vinculantes, para tras el fallo ser desarrolladas por otros profesionales distintos a sus autores, concursos en los que se condiciona la partilipación a citterlos localistas. como tener despacho dentro de ámbios limilados; 0 a hecho de haber realzado antriorm Salvo ecońmica cquivalonte, elc.

Salve y anónimo, todas las demás versiones encierran como ya hemos apuntado han nacido a lo largo de la histori de la arquitectura por el temor de los convocantes de poner en manos de pravitectos desconocidos o ineficaces a respon mabilided de desarrollar proyectos importantes, por no hablir de una desconfianza hacia los jurados a los que paradólcamente elige para deliberar Entran aqú en conficto la esencia de un concurso de ideas con la oblijacín lega de convocar concursos. Cunto may os el nivel de gencia de requistos en la restrición de los paticipantes, el organizador se a eja de la idea de concurso y por conla encargo directo.

Peter Murray lo expresa abiertamente de este modo n la introducción de su interesante libro sobre la Ópera de Sídney: "Sibión de su interante libro sobre la Ópera tunidad de descubrir nuevos talentos, se corre el riesgo seleccionar proyectos que verdaderamente el cliente no

7. Murray, Peter: The saga of Sydney Opera House: the dramatic story of the design and construction of the icon of modern Australia. London [etc.]: Spon Press, really want an architect who can design but cannot build and building that does not work. The judding process throws up designn that are visually and arch tectural striking - and often ignores the more reticent entries" (...) "As a direct result of the Sydney Opera House, open competitions have been used less and

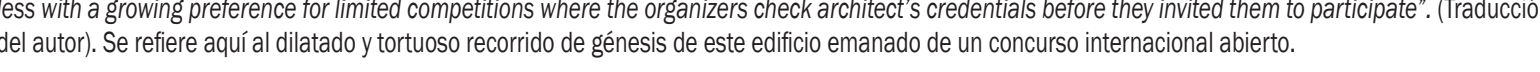

desea, arquitectos que saben diseñar pero no construir, y (C) (Is más conse y fecuentemente ignora aquellas propues(l...) "Como consecuencia directa tos han sido palatinama de Sianey, los concursos abierho una creciente preferencia los concursos restringidos los arquitectos antes de invitarlos a participar"7

UNA ARQUITECTURA PARA LOS CONCURSOS "Los arquitectos sabemos que es muy distinto trabajar con un cliente especifico, con un encargo cerrado, que sempre excis a innonón y a inestio, que creo que entendemos formas diferentes de abordar la actived proyectual". Luis Fernández-Galiano.

Sobre la capacidad de suscitar la investigación que

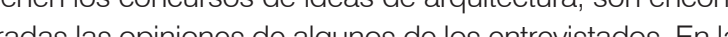
medida en que cliente está temporalmente ausentey se

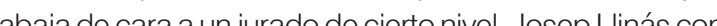
. -

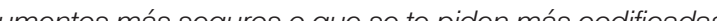
pamónFernández Alonso dice creer en "el proyecto como proceso de investigación Os concursos desempeñan aquí un papel muy importanle ysino existieran habrí que inventarlos porque permiten al rouitecto un espacio de libertad y lo garantizan. El concurso es la herramienta que más identifica la investigación

Sin embargo hay voces críticas con la identificación mode modo: "Yo no estaría muy seguro de la relación divecta ente elificios que no funcionan. El proceso de selección del judonde los organizadores contrastan las credenciingidos 
nuevos temas y siven para desarrollar ciertas ideas o planleamientos anteriores, que dificillmente se plasman en un encargo directo por la dificultad de encontrar un cliente abierto a diferentes alternativas. Pero el concurso tiene normalmente un problema y es el (breve plazo de) tiempo: poco se puede experimentar en un sentido cierto. La investigación necesita mucho tiempo".

El posicionamiento intelectual del arquitecto ante el reto de resolver un ejercicio libre en un contexto competitivo, puede llevarle al divorcio entre una idealización de esa arquitectura y su capacidad para materializarla, a veces con un indisimulable desconocimiento de la técnica que las hace viables o del coste económico y temporal de su ejecución. Encontramos así casos paradigmáticos como lo fue el citado Palacio de la Ópera de Sídney del danés Jørn Utzon', u de Zaha Hadid para la expo-2008 de Zaragoza"; La Cidade de Zaha Hadil para la expo-2008 de Zaragozá, La Cidade da Cultura de Santiago de Compostela, concurso ganado por el prestigioso arquilecto Peter Ensenman en 1999, 0 el Metropol-Parasol de Jurgen Mayer en Sevilla de 2004 contectación ciudadana y pol'tínado, con una importante ces apazada entrega de una ces aplazada entrasa de una obra con imponantes desvia-

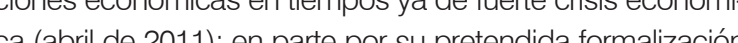
ca (abin telizada en un casco histórico muy blindación

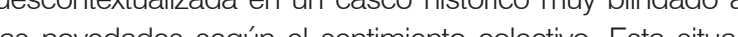
ción contatable en cél sen conto colcolivo. Esta siluacion constarsos de arquitectura y con ellos a los propios ar unectos, jurados y organizadores, a altas cotas de impopulectos, Juadosy organiza yoes, a allas colas de impo-

Por orra part y desde un punto de vista disciplinar, sería pérdida de autonomía a

que el arquitecto se somete de forma voluntaria en este ejercicio de lo imposible que supone la denominada popuabandono parcial de su responsabilidad en aras de concenfrar una investigación descompensada hacia los aspectos más formales de la arquitectura, quedando depositada la viabilidady veracidad de su concrecion material en manos de potentes despachos de ingeniería que posteriormente salvan las ideas surgidas de este celo competitivo e innovador. En ficar notables destiguraciones de las ideas originales; en cas los casos, elevadas desviaciones presupuestarias. A propósito de la cuestionable verosimilitud de muchas propuestas, llevadas a concurso con imágenes muy sugerentes, el arquitecto Javier Terrados afirma admirar "la capacidad de algunos estudios de presentar un producto gráfico miablidad de la propuesta (...). . De nuevo surge la polémica como consecuencia de una aparente frivolidad de los concursantes, pero tambiên por la supuesta falta de rigor posita posta la responsabilidad de elegir las propuestas. Estos, a de de debeŕn sobre las consecuencias ecońm na lama da de áención sobre las consecuencias económicas y de

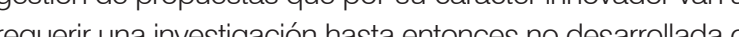
predeciblemente costosa.

LOS CONCURSOS DE ARQUITECTURA Y EL TRABAJO NO REMUNERADO

"Esta es quizás la parte más débildel proceso, porque hay un esfuerzo muy grande por parte de los profesionales que en cierto modo, salvo los premiados, no ven equilibrada la respuesta. Hay concursantes y hay ideas que son brillantes yno adquieren el reconocimiento que debieran". María Dolores ocasiones este ajuste a la realidad constructiva llega a signimuy atracivo que convence y compensa las carencias de

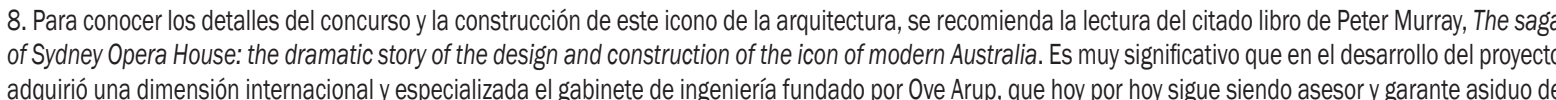
arquitectos concursantes en todo el mundo. El edificio tardó en construirise 18 años, inauguránndose en 1973, y su presupuesto superó el $1.450^{\circ} 00 \%$ del coste nicialmente calculado en la propuesta de concurso de 1957.

9. El número especial sobre la Exposición Internacional le Zaragozaz 2008, de jullio de 2008 de la revista Europ'A, publicación de la Asociación para la Promoción Técnica del Acerro, comenta en una serie de articullos unas Jornadas técnicas celebradas en abril de ese año en la sede de la Confederación Hidrográrítica a buen término un proyecto complejisimo que, en principio, carecía de concepto, al menos en lo que a solución estructural se refería, por lo que hubo que pensarlo y definirlo a posteriori (...)" En el mismo articullo se revelan "los cambios que se introdujeron en la propuesta inicial de ARUP (un cajón de hormigón realizando FHECOR una variante (un cajón de acero que luegog derivé en la actual celosía (...)", o más adelante relatando cómo la ubicación de los "hueccos
definitivos que se han abierto en el puente" están supeditados a cálculos iterativos de la estructura.
Gil Pérez, Jefa del Servicio de Arquitectura de la Consejería de Obras Públicas y Transporte de la Junta de Andalucía Tratamos finalmenteelaspectomásnegativodelosque pueden ser abordados al hablar de los concursos de arquiectura, sobre todo de los concursos de ideas o proyectos Si atendemos a que el objeto genuino de la profesión del arquitecto es el proyecto de arquitectura, en toda su dmensión, como representación de la misma, como prototipo y modelo verificable y como partitura para su construcción en un lugar y tiempo concretos, resulta dificil comprender que los arquitectos esten dispuestos a responder una y otra vez de manera masiva ei hiensa a concursos por lo genera no remunerados, anticipando gran parte de su trabajo a posible obtención de un encargo. Las condiciones de incer tidumbre extremas de los concursos, en los que el acierto queda muy condicionado a variables que no son controlables sôlo por la calidad del proyecto, y con bajas probabildades de exito en función del normalmente alto número de

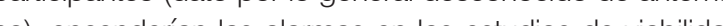
no), encenderían las alarmas en los estudios de viabilida

Según el arquitecto Patxi Mangado, "A los arquitectos se nos puede demandar por muchos errores, pero no es el de so. Sin embergo cada vez más, nos progunamos siesta . cetorias bien planteadas, sufiointemente compensalas $y$ con jurados de calidad contrastada. (..) Cada vez más los concursos de arquitectura y por supuesto hay excepciones están marcados por unas maneras que atentan contra b digninguneado o infravalorado (...)"12

Para los no ganadores, el concurso representa como mucho algún tipo de enriquecimiento profesional íntimo. As lo manifiesta Ramón Fernández-Alonso: "Dentro de la producción de un arquitecto, los concursos no ganados están parcialmente. Esos concursos no ganados son peldaños en los que uno se apoya para en un determinado momento dar un acierto y llegar a un determinado sitio". Con esta opinión coinciden la mayoria de los entrevistados, defendiendo e concurso como oportunidad para el arquitecto de ejercitar se en terrenos que de otro modo son difíciles de explorar, el propio Peter Murray lo explica en parecidos términos en la introducción del libro referido anteriormente: "Los concursos demandan inmensas cantidades de horas no remuneradas por parte del arquitecto. A pesar de lo cual, una buena parte de la pracica de los estuclos está dispuesta a emplear el liempo en ellos, no solo por la oportunidad de ganarlos, sino lambien para comprobar sus ideas arquitectónicas, manteCabra ajur ans recursos humanos

Cabria analizar aunque sea brevemente la estructura produciva y los esquemas de funcionamienio internos de los estudios de arquilectura asiduos a los concursos. Nor-

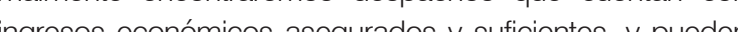
ingresos económicos asegurados y suificientes, y pueden suragio de costosas ontregas, par in poner en peligro su viabilidad profesional Por otra parte en nuren polyo su viablicad profesional. Mor ora pare de recambio, cualficados sobre todo en el uso de potentes herramientas inforícicas, y capaces por tanto de genera mácenes atroctivas con yelitiv rapidez a partir de ideas báscas muy preliminares en el proceso proyectual: mano de obra que acepta trabajar gratis o casi gratis con la única contrapartida de adquir prestigio curricular por perticipar bajo la firma del estudio, y a promesa de ver reconpensado económicaylaboralmentesuesturzo sól enelcasode ganar el encargo lo cual constituye un estím ul añadido Un "intipor serí el de un equipo regentado por arquectos que ejercen

10. Es conocido que el arquitecto Wilfried Wang, frente a la defensa que encabezó su colega Luis Fernández-Galliano, sostuvo una posición contraria a este

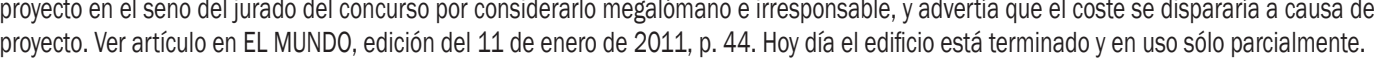
S.L. pp. 8-23.

12. Patxi Mangado, Concursos de arquitectos o chapuzas, EL PAíS, edición de 28 de febrero de 2009, p. 40.

13. Murray, Peter: The sagá of Sydney Opera House: the dramatic story of the design and construction of the icon of modern Australia. London [etc.]: Spon Press, 2004, p.xvii: “The system also demands a huge number of unpaid man-hours on the part of the architect. Despite this, many architectural practices are willing to expend the time, not just to winning, but to test their architectural ideas, to keep their eye in and to develop their staff'" (Traducción del lautor). 
para estas tareas a estudiantes y arquitectos recién licenciados y previamente seleccionados por su capacidad y dispuestos a desplegar lo mejor de sí mismos con illusión y entrega sin reservas.

La prolifica producción de ideas y la espectacular puesta en escena a que dan lugar los concursos, devaIuan la percepcion externa del trabajo de los arquitectos. Las administraciones convocantes y el gran público llegan a creer, seducidos por la abundancia y calidad de los proyectos presentados, que estos se gestan con bajo coste y escasa dedicación, y que las ideas surgen de una suerte de técnicas automalizadas que no precisan especial esfuerzo. De otra manerano seria comprensible que un profesional altamente cualificado trabaje con tanto compromiso yenerosidad. Pero lo cierto es que los concursos consumen cuantiosos recursos econónicos y suponen elevados sacícios personales para los concursantes. Carmen . mbenón comenta de modo expresivo su experiencla en UROPAN. La verdad es que cuando (desde el jurado) se

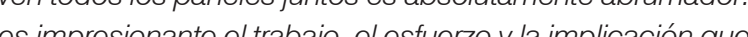
hay detrás Produce desazón ver que de todos se save

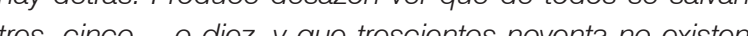
más. sobre todo cuando entre estos hay propuestas muy valorables a marcen de los premiodos".

Resultaría perverso que la competitividad fuese admitida aprovechada por los promotores de concursos como palanca para explotar especulativamente la cantidad y calidad riente ideó́ócica apunta sin embergo en es a digur hora de definir los nuevos modelos de recciones laborales: "Quienes trabaian gratis tienen más ambición más hambre que aquellos que perciben un sueldo. Y además son más creativos. (.) No se trata de pasantís, précticas o júnes ecién licenciados Hablamos de profesionales más omenos cualificados"15. En otras disciplinas existen frentes de opinión

en los que, jugando con la superabundancia de profesioname más. Son los movimientos Gratis No 1 rabajo $0^{16} \mathrm{en}$ el mundo del periodismo, o el más antiguo e internacional NO.SPEC en el ámbito del diseño grá́ico y los concursos del ramo Antonio Ortiz es rotundo y acertado en este asunto el sistema de concursos es económicamente disparatado: para elegir un proyecto, recibir doscientos... Calculas el número de horas y el estuerzo y el valor económico de lo que está alli, y es un disparate propio de sociedades que podemos llamar opulentas".

Y Rem Koolhaas, a quien precisamente no le han ido mal las cosas en las más selectas competiciones restringidas, atrentado por el desarrollo del concurso en San Petersburgo para la sede de Gazprom, lanzo en enero de 2007 unam "Estamos peran undo que los concursos internacionales

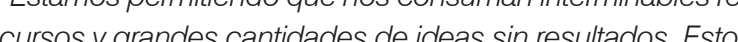
cursos y grandes cantidades de ideas sin resullados. Estoy

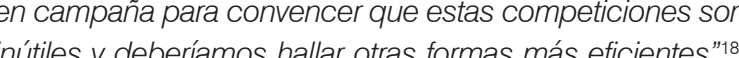
Esta es sin duda la gan deda que los promon Esta es sin duda la gran deuda que los promolores de contrar fómulas que hagan de la profesion. la de en-

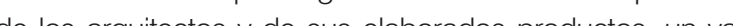
lory no un desecho Habrí que repensa los concursos de lo esinu pelexín que la elección de una propuesta es siempre por comperación entre las alternativas presenadas. Desde esta perspectiva las no elegidas juegan elinsustituble papel de fijer te exigente frontera a jun despunta b solucín premiable yeste valo dabe reveliren sus creadors en vez de ent pobrecelos Y Yuiźs tambín os arquitectos deberímos abandorer nuestra ingenua at locomplacencia en la defensa de los concursos, que ahora aún más que antes se torna lejos de la relidad y por una vez de forma solidaria exigir fórmulas más justas, equilibra

4. Exposición Concursos de Arquitectura con Participación de Jurado. 2002/2006; CD catálogo de la exposición. Sevilla, enero 2008. En este catálogo se puede contabilizar que a los 29 concursos expuestos se presentaron en total 1.802 proyectos.

15. Fernández, Tino. Trabajar sin sueldo, la nueva realidad. Expansión.com. Expansión y empleo [en linea]. 21-04-2011. Disponible en Internet: http://Wwu. expansion.com/2011/04/21/emplee/desarrollo-de-carrera/1303385156.hth

6. http://www.facebook.com/pages/Gratis-No-Trabajo/22815761721149

17. http://Www.no-spec.com//

\section{Bibliografía}

Murray, Peter: The saga of Sydney Opera House: the dramatic story of the design and construction of the icon of modern Australia. London [etc.

Molina, Carlos: Arquitectura participativa. En Hechos, noticias, actualidad. Mayo 2007. № 21. Madrid: Hermandad Nacional de Arquitectos. pp. $42-46$ Pastor Segovia, Ignacio; Tejido Jiménez, Javier: Conversación con Javier Terrados. En Andén, revista de arquitectura. 2011. № 1.. Sevilla: Signos Visibles S.L. p. 8-23.

Villanueva Cajide, Beatriz; Casas Cobo, Francisco Javier: Catedral Fluvial. Pabellón Puente sobre el río Ebro. En Europ'A. Julio de 2008, No especia

De La Iglesia Salagado, ,élix; Moreno Pérez, José Ramón: Por una ecologomía arquitectónica: Ios concurros como dispositivo de regulación de la octubre 2006. pp. 41-55.

Rojo Carrero, Jesus: Trillo Martínez, Valentín: Catálogó de la Exposicición Concursos de Arquitectura con Participación de Jurado. 2002/2006: [CDROM]. PC Pentium III o superior. Sevilla: Consejería de Obras Públicas y Transporte de la Junta de Andalucía, enero 2008.

Rojo Carrero, Jesuss: Catâlogo de la Exposición 13 Concursos de Arquitectura con Participación de Jurado. Retrospectiva 1998/2001; [CD-ROM]. PC Pentium II o superior. Sevilla: Consejería de Obras Públicas y Transporte de la Junta de Andalucía, octubre 2002.
Jesús Rojo Carrero, (Sevilla, 1963), arquitecto (1989) por la ETSA Sevilla. Como Secretario Técnico ha desarrollado varios con

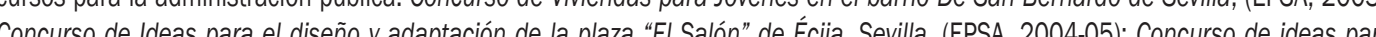
rehabilitación del Ayuntamiento de Éciaja, Sevilla, (COPT, Junta de Andalucía, 2006): Concurso de ideas de 245 VPO en EI Porveni Sevilla, (EMVISESA, 2006-07). Elaboración de la documentación técnica para la convocatoria de concurso para la adiudicación de fa consultoría y asistencia técnica de la Rehabilitación y ampliación del edificio del Ayuntamiento de Fuentes de Andalucía, (COPT, Juntra de Andalucía, 2004). Ha sido Comisario de la Exposición 13 Concursos de Arquitectura con participación de jurado. Rretrospectiva 98-01, (COPT, Junta de Andalucía. (Sevilla, octubre de 2002; Córdoba, enero de 2003; Jaén, abril de 2003; Granada, enero 2004) Exposición Concursos de Arquitectura con participación de jurados, CPAJ 2002/2006. (En colaboración, Valentín Trillo Martínez, COPT, Junta de Andalucia. (Sevilla enero de 2008; Granada, noviembre de 2008, Quito - Ecuador, noviembre de 2010). Miembro de Jurado de los X Premios De Arquitectura 1997. COAAOc; del Concurso de Ideas para la i implantación de un aparcamiento subterráneo yordenación de la Plaza del Ayuntamiento de Marchena, Sevilla (1999), (COPT, Junta de Andalucía). 


\section{Autor imagen y fuente bibliográfica de procedencia}

Información facilitada por los autores de los artículos: página 20, 1 (AA.W.: Concurso de Proyectos 1996. Propuestas de los equipos adjudicatarios. Sevilla: Dirección General de Arquitectura y Vivienda, Junta Andalucía, 1997, portada), 2 (Dirección General de Arquitectura y Vivienda, Junta Andalucía, tríptico); página 22, 3 (Manuel Toledo), 4 (Andrés López); página 23, 5 y 6 (Jesús Granada) 24, 7 (Fernando Alada), 8 (Jesús Granada), 9 (Fernando Alda); páginas 42, 43 y 44, 1 a 3 (El Croquis n53 0MA/Rem Koolhaas. Madrid: El Croquis, 1994, pp. 73 y 78); páginas 46, 47, 4 a 6 (AA.VV: Exposición Universal Sevilla 1992: ideas para una ordenación del recinto, Sevilla: Expo 92, Comisaría General de España, 1986); página 49, 7 (Ceccarelli, P. La construcción de la ciudad soviética. Barcelona: Gustavo Gili, S.A., 1972, figura 26, Anexo ilustraciones, p. XI), 8 (De Michelis, M.; Pasini, E. La Citta`Sovietica 1925-1937, Venecia: Marsilio Editori, 1976, p. 80); páginas 50y 51, 9 y 10 (9. De Michelis, M.; Pasini, E. La Citta 'Sovietica 1925-1937, Venecia: Marsilio Editori, 1976, pp. 81y 180); página 55,1 (Reproducido de Deutches Dokumentation fur Kunstgeschichte, Universitat Marburg, en www.fotomarburg.de); página 56, 2 (Cristina Gastón Guirao); página 57, 3 (Der Scherei nach den Turmhaus. Der IdeenwettbewerbHochhaus am Bahnhof Friedrichstrasse. Berlin 1921/22. Berlín: Argon Verlag GmbH, 1988, p. 39), 4 (Cristina Gastón Guirao en base a Der Scherei nach den Turmhaus. Der IdeenwettbewerbHochhaus am Bahnhof Friedrichstrasse. Berlin 1921/22. Berlín: Argon Verlag GmbH, 1988, p. 38); página 58, 5 (AAVV. Mies in Berlin. (publicado en relación con la exposición del mismo nombre). New York/Berlín: MOMA, 2001, p. 183); página 59, 6 (Reuter, Helmut; Schulte, Birgit. Mies and modern living. Interiors, furniture y photography. Ostfildern: Hatje Canz Verlag, 2008, p. 50, 210 y AAVV. Mies in Berlin. (publicado en relación con la exposición del mismo nombre). New York/Berlín: MOMA, 2001, p.181), 7 (AAVV. Mies in Berlin. (publicado en relación con la exposición del mismo nombre). New York/Berlín: MOMA, 2001, p. 182); página 60, 8 (Cristina Gastón Guirao en base a Der Scherei nach den Turmhaus. Der IdeenwettbewerbHochhaus am Bahnhof Friedrichstrasse. Berlin 1921/22. Berlin: Argon Verlag GmbH, 1988, pp. 45, 105); página 62, 9 y 10 (Reuter, Helmut; Schulte, Birgit. Mies and modern living. Interiors, furniture y photography. Ostfildern: Hatje Canz Verlag, 2008, pp. 230, 252, 258); página 64, 11 (Zevi, Bruno (ed): Erich Mendelsohn. Opera Completa. Turín: Testo \& Immagine, 1997, p. 197), 12 (Der Scherei nach den Turmhaus. Der IdeenwettbewerbHochhaus am Bahnhof Friedrichstrasse. Berlin 1921/22. Berlín: Argon Verlag GmbH, 1988, pp. 181, 184); página 65, 13 (Schulze, Franz. Mies van der Rohe. Una biografía crítica. Madrid: Hermann Blume, 1986 p. 155); página 69, 1 (Inv. Nr. HP 041,005. Architekturmuseum der Technischen Universität Berlin in der Universitätsbibliothek); páginas 70, 72, 73, 74, 2 a 5 (@FLC/Vegap, Sevilla, 2012. @Pierre Jeanneret, Vegap, Sevilla, 2012), 6 (Theaterwissenschaftliche Sammlung Universität zu Köln); página 75, 7 y 8 (Inv. Nr. 2750, Inv. Nr. F 1604. Architekturmuseum der Technischen Universität Berlin in der Universitätsbibliothek); páginas 76 y 80, 9 a 11 (@FLC/Vegap, Sevilla, 2012. @Alexander Rodchenko, Vegap, Sevilla, 2012), 12 (0148126. Tretyakov State Gallery, Moscow); página 82, 13 (Austrian Frederick and Lillian Kiesler Private Foundation Vienna), 14 ((СFLC/Vegap, Sevilla, 2012. @Pierre Jeanneret, Vegap, Sevilla, 2012); página 84, 15 (@FLC/Vegap, Sevilla, 2012. @Pierre Jeanneret, Vegap, Sevilla, 2012), 16 (X1119.3a-c. Colletion of Brooklyn Museum, New York); página, 85, 17 (Cabinet Ejzenstejn, Moscow (Hayuнoмемориальный кабинет-музей С. М. Эйзенштейна), 18 (Imagen de dominio público. http://commons.wikimedia.org/wiki/File:Sadanji_Ichikawa_Il_and_Sergei_Eisenstein. jpg); páginas 92 y 94, 1 y 2 (Roth, Alfred: La nouvelle architecture presentée en 20 exemples, Die neue architektur dargestellt an 20 Beispielen, The new architecture presented in 20 examples. 1930-1940. Zúrich: Verlag für Architektur Artemis, 1975, pp. 131, 132); página 94, 3 (Architettura, № XVI, Fasc. IX. Milán: septiembre de 1938, p. 574); página 96, 4 (Roth, Alfred: La nouvelle architecture presentée en 20 exemples, Die neue architektur dargestellt an 20 Beispielen, The new architecture presented in 20 examples. 1930-1940. Zúrich: Verlag für Architektur Artemis, 1975, p. 136), 5 (Fondazione Giuseppe Terragni; reproducida en Curtis, William J. R.: La arquitectura moderna desde 1900. Londres: Phaidon, 2006, p. 366); página 97, 6 (International Institute of Social History, Amsterdam; reproducida en Molema, Jan: Jan Duiker. Barcelona: Gustavo Gili, 1996, p. 85.), 7 (Emilio Cachorro Fernández); página 98, 8 y 10 (Roth, Alfred: La nouvelle architecture presentée en 20 exemples, Die neue architektur dargestellt an 20 Beispielen, The new architecture presented in 20 examples. 1930-1940. Zúrich: Verlag für Architektur Artemis, 1975, p. 137), 9 (AA.W.: Iǵnazio Gardella, 1905-1999. Arquitectura a través de un siǵlo. Catálogo de la exposición. Madrid: Ministerio de Fomento, 1999, p. 13); página 100, 11 y 12; página 102, 13 (Maffioletti, Serena: BBPR arquitectura. Banfi, Belgiojoso, Peressutti, Rogers. Sevilla: COAAO, 1996, pp. 47, 56, 67), 14 (Musée du Petit Palais, Ginebra; reproducida en Stoichita, Victor l.: Ver y no ver. Madrid: Siruela, 2005, p. 38); página 108, 1 (Arquitectura. Agosto 1969, № 128. Madrid: Colegio Oficial de Arquitectos de Madrid, 1969. Primera página; Nueva forma: arquitectura, urbanismo, diseño, ambientes, arte. "Concurso Universidad Autónoma de Madrid". Septiembre 1969. № 44. Madrid, 1969. Portada; Nueva forma: arquitectura, urbanismo, diseño, ambientes, arte. "Concurso Universidades Autónomas de Bilbao y Barcelona". Enero 1970. № 48. Madrid, 1970. Portada); página 110, 2 (Nueva forma. Septiembre 1969. № 44. cit. p. 5); página 111, 3 y 4 (Arquitectura. Agosto 1969, № 128. cit. pp. 33 y 35); páginas 112 y 113, 5 a 9 (Nueva forma. Septiembre 1969. № 44. cit. pp. 33, 30, 81, 18, 24); páginas 114, 115 y 116, 10 a 14 (Nueva forma. Enero 1970. № 48. cit. pp. 23, 20, 56, 69, 96), 15 y 16 (Cuadernos de arquitectura. Primer trimestre 1970. № 75. Barcelona: Colegio Oficial de Arquitectos de Catalunya, 1970. pp. 58 y 66); página 117, 17 (Nueva forma. Enero 1970. № 48. cit. p. 31); página 118, 18 (Nueva forma. Septiembre $1969 . N^{\circ} 44$. cit. p. 4; Temas de arquitectura y urbanismo. Septiembre 1969. № 123. Madrid-Barcelona, 1969. p. 5); página 123, 1 y 2 (Bucci, Ângelo; Puntoni, Álvaro; Vilela, José Oswaldo. Foto: Nelson Kon.); página 126, 3 (Parada, Sérgio Roberto. Projeto cedido por Rodrigo Biavati), 4 (Gorgati, Vinicius; Franco, Fernando de Mello; Moreira, Marta; Braga, Milton. Projeto cedido pelos autores), 5 (Rodrigues, Sidney Meleiros; Saraiva, Pedro Paulo de Melo; Rosemberg, Marcelo; Vaisman, Jacobina; Lobo, Marcos Toledo; Nunes, Ronaldo Soares.Projeto cedido pelos autores); páginas 128, 129, 6 a 12 (Bucci, Ângelo; Puntoni, Álvaro; Vilela, José Oswaldo. Projeto cedido pelos autores); página 131, 13 y 14 (Bucci, Ângelo; Puntoni, Álvaro; Vilela, José Oswaldo. Foto: Nelson Kon); página 136, 1 (Fromonot, Françoise. En Jørn Utzon, architetto della Sydney Opera House. Milano: Electa, Documenti di Architettura, 1998. pp. 15), 2 (Piano, Renzo. En Giornale di Bordo. Florencia: Passigli Editori, 1997, pag 38); página 138, 3 (Mikami, Yuzo. En Utzon's sphere. Sydney Opera House. How it was designed and built.Tokio: Ed. Shoku Kusha, 2001), 4 (Silver, Nathan, The making of Beuabourg, A building biography of the Centre Pomidou, Paris. Cambridge, Massachussets: Ed The MIT press, 1994); página 139, 5 (Piano, Renzo, Renzo Piano Building Workshop, complete works. Londres: Phaidon Press Limited, 1993. vol 1. pp. 54), 6 (Weston, Richard: Utzon : inspiration, vision, architecture. Hellerup (Dinamarca): Editorial Blondal, , 2002. pp. 117); página 140, 7 (Weston, Richard: Utzon: inspiration, vision, architecture. Hellerup (Dinamarca): Editorial Blondal, , 2002. pp 116), 8 (Fromonot, Françoise. En Jørn Utzon, architetto della Sydney Opera House. Milano: Electa, Documenti di Architettura, 1998. pp 64), 9 (Ferrer, Jaime, Jørn Utzon. Obras y proyectos. Barcelona: Editorial. Gustavo Gili, 2008. pp. 159); página 10, (Rice, Peter: "La structure métallique". En Architecture d'aujourd'hui. Febrero 1982, n 219. pp. 62. París: Editorial Groupe Expansion,1930), 11 (Piano, Renzo, Renzo Piano Building Workshop, complete works. Londres: Phaidon Press Limited, 1993. vol 1. pp. 45); página 142, 12 (Architectural Design', vol 47 nº 2, febrero 1977); página 144, 13 (Weston, Richard: Utzon : inspiration, vision, architecture. Hellerup (Dinamarca): Editorial Blondal, 2002); página 148, 1 (AA.W.: Arquitectura Viva. "Obras singulares". 1998, № 62. Madrid: Arquitectura Viva. 1998. pp. 78-81); página 149, 2 y 3 (AA.W.: Concurso 2G competition: Parque de la Laguna de Venecia = Venice Laggon Park. Barcelona: Gustavo Gill, 2008. pp. 81, 13); página 150, 4 (Jacopo de 'Barbari, [Web en linea]. [Consulta: 04-10-2012]), 5 (Zumthor, Peter: Atmósferas: entornos arquitectónicos, las cosas a mi alrededor. Barcelona: Gustavo Gili, 2006. p. 6); página 151, 6 (David Chipperfield 2006-2009. El Croquis. № 120. Madrid: El Croquis. 2008. p. 66); páginas 152, 153,7 a 9 (AA.W.: Carlos Ferrater. Works and Projects 1980-2000. Barcelona: Actar, 1998); páginas 154, 155, 10 a 12 (Enric Miralles + Benedetta Tagliabue: 1995-2000. El Croquis. № 72. Madrid: El Croquis. 2000. pp. 132-141); página 156, 13 a 15 (David Chipperfield 2006-2009. El Croquis. № 120. Madrid: El Croquis. 2008. pp. 64, 67, 66); página 157, 16,17 (Luca Nicolao Photography, [Web en linea]. [Consulta: 19-9-2012]), 18 (David Chipperfield 2006-2009. El Croquis. № 120. Madrid: El Croquis. 2006. p. 66); páginas 162, 164, 167, 169, 170, 1 a 6 (๑ F.L.C. /VEGAP, Sevilla, 2012. OPierre Jeanneret, VEGAP, Sevilla, 2012); página 176, 1 (García Mercadal y S. Giedion. Congresos internacionales de arquitectura moderna. AC. №5. Primer Trimestre de 1935. Barcelona. GATEPAC.1932 p.12); páginas 178, 179, 2 a 4 (AC. №11. Tercer trimestre de 1933. Barcelona. GATEPAC. 1933. pp. 37, 34, 35); página 180, 5 (Le Corbusier et Pierre Jeanneret : Ouvre Complète 1910-1929. Zurich : Les Édtions D’Architecture. 1964 p.65), 6 (AC. NN11. Tercer trimestre de 1933. Barcelona. GATEPAC. 1933. p. 34), 7 (Le Corbusier et Pierre Jeanneret: Ouvre Complète 1910-1929. Zurich : Les Édtions D’Architecture. 1964 p.65), 8 (L'Habitation minimun. Frankfurt. 1929. Zaragoza: Edición facsimil Colegio de Arquitectos de Aragón.1997.p.26), páginas 181, 182 (AC. №11. Tercer trimestre de 1933. Barcelona. GATEPAC. 1933. p. 37, 1); páginas 189, 190, 1 y 2 (Cássia de Souza Mota); página 191, 3 (Boero, Dolores; Castellote, Ana Maria; Puglisi, Jose Agustin. Fonte: http://www.uia-architectes.org/texte/ england/Celebration/SPrix/Reglll/Frames.html), 4 (Fernandez, Alberto; Guerrero, Camilo; Javiera, Paulina; Sanchez Recio, José Manuel. Fonte: http://www.celebcities2.org/); página 192, 5 y 6 (Nikita, Sergienko. Fonte: http://www.celebcities3.org/wc/content/18/pdf/054FECC2-C9DC-410A-A044-CB2A0BBD25EB.pdf) 